\title{
Mobile Based User-Centered Learning Environment for Adult Absolute Illiterates
}

\author{
Inayat ur-Rehman, ${ }^{1}$ Azra Shamim, ${ }^{1}$ Tamim Ahmed Khan, ${ }^{2}$ \\ Manzoor Elahi, ${ }^{1}$ and Sajjad Mohsin ${ }^{1}$ \\ ${ }^{1}$ Department of Computer Science, COMSATS Institute of Information Technology, Islamabad 45550, Pakistan \\ ${ }^{2}$ Department of Software Engineering, Bahria University, Islamabad 44000, Pakistan
}

Correspondence should be addressed to Tamim Ahmed Khan; tamim@bui.edu.pk

Received 27 October 2015; Accepted 11 January 2016

Academic Editor: Miltiadis D. Lytras

\begin{abstract}
Copyright (C) 2016 Inayat ur-Rehman et al. This is an open access article distributed under the Creative Commons Attribution License, which permits unrestricted use, distribution, and reproduction in any medium, provided the original work is properly cited.

Education plays a vital role in the success of any community. Countries with increased literacy rate have improved their status on the world map. In recent years, the use of e-learning methodologies has been significant. However, majority of the previous methodologies are focused on the formal education or toddlers. The technoliteracy solutions for children are not suitable for adults and those designed specifically for adults are text dominant and require the users of these applications to be functional literate. Moreover, users' interest (sense of belonging) is not taken into consideration in existing solutions. To address the aforementioned issues, a user study is conducted to collect users' interests. Another highlight of our study is that we develop our system as a mobile device application to facilitate our target user group. Based on the collected interests, a 3D virtual learning environment is designed and developed for adult illiterate learners. To evaluate the effectiveness of the proposed environment, an experimental study is carried out with users. The results show that the proposed learning environment significantly improves adults learning.
\end{abstract}

\section{Introduction}

Illiteracy is one of the challenges that modern world faces today and around more than 770 million adults are illiterates [1]. UNESCO has adopted an indirect policy to tackle the issue where adult education is deemed necessary for the sake of present and future generation [2]. It is worth mentioning that China, India, Egypt, Ethiopia, Indonesia, Bangladesh, Nigeria, and Pakistan house more than sixty percent of illiterate population of the world [3]. Director General UNESCO says, “...literacy needs everywhere are changing and must be addressed in innovative ways" [4]. The statement raises the question, what are these innovative ways? Of course, these innovative ways have many facets; one of them is technology.

Information and Communication Technology (ICT) has provided potentially a valuable teaching aid and has brought a revolution in the field of education [5]. This has compelled educationists to involve ICT in education to improve effectiveness of teaching and learning outcomes [6]. Video conferencing, virtual learning environments, interactive white boards, and other such interactive technologies have been extensively adopted by education institutions. For more effective delivery of learning contents, trends are changing from text dominant material to two-dimensional (2D) or three-dimensional (3D) materials. Recently, educationists have diverted their attention to $3 \mathrm{D}$ virtual environments as these environments have great potential to imitate culture and context and also emulate experiences very close to real life with little or no risks in a cost effective way which makes it more appropriate for learning than the orthodox $2 \mathrm{D}$ applications $[7,8]$. Moreover, 3D virtual environment is helpful to enhance socialization, collaboration, communication, and experimental learning.

Although technology is being used for main stream education, it is surprising to note that technology-assisted solutions for adult illiterates are not available in the first place. If available, they target functional illiterates (a person who can read and write basic sentences) only. Little attention is paid on technology-assisted basic educational environment for absolute illiterates (persons who cannot read and write 
basic sentences). 3D virtual environment can be designed as learning platform in order to reduce the text dominant interfaces so that the issues pertaining to the use of technology could be addressed. Although few learning environments were designed for absolute illiterates, no one considered the aspect of "sense of belonging" that defines acceptance as a member or part. In this paper, we design and develop a 3D virtual learning environment to teach numeracy education to absolute illiterate adults by integrating the sense of belonging in the environment. We develop and test a user-centered approach by bringing users into the design process to ensure that the environment meets their needs and desires.

In this paper, our focus is to design and evaluate a $3 \mathrm{D}$ learning environment for adult absolute illiterates (people who are unable to read or write the numbers). Firstly, interests and likings of users are collected by conducting a user study to design a $3 \mathrm{D}$ environment for adult illiterate. Secondly, based on the collected data, a users' centered 3D learning environment is designed and developed for adult absolute illiterates for numeracy education. Thirdly, experiments with users are conducted to evaluate the effectiveness of the proposed learning environment. Specifically, their types of learning environments, (i) general environment (without sense of belonging), (ii) environment for farmers (with sense of belonging), and (iii) environments for women (with sense of belonging), are developed in this study. Adult learning efficiency on environments incorporating sense of belonging (farmer and women environments) is compared with general environment.

The remainder of this paper is organized as follows. Section 2 describes existing work on sense of belonging and learning environment. Methodology adopted in this work is explained in Section 3. Section 4 introduces the proposed 3D learning environment. This is then followed by Section 5 that presents the evaluation of the proposed environment, and finally Section 6 concludes this research work.

\section{Related Work}

Different virtual learning environments are developed to enhance the learning process. A language learning tool called Physically Interactive Learning Environment (PILE) is developed for English language and, subsequently, a study regarding the effectiveness of this environment is reported [10]. The environment is developed using a personal computer, a webcam, and a projector. The results of an empirical study revealed that PILE increases students' learning motivation and teachers' satisfaction. An e-course implemented in the Second Life 3D virtual world is developed in [11] to provide technology enhanced learning. To increase mathematical skills of deaf children, a 3D learning environment is developed [12]. The environment provided better learning than existing virtual learning environments. Similarly, the impact of virtual reality learning environments to elementary numeracy education using $3 \mathrm{D}$ virtual manipulatives is investigated and provided set of benchmarks/guidance for development of virtual learning environments [13].
In the context of game-based learning environment, [14] presented the design of a learning environment called Crystal Island: Uncharted Discovery for science students. Their findings showed that the environment improved participation, problem solving skills, and knowledge of both male and female students. Similarly, a collaborative game-based learning environment is developed by [15] by amalgamating a grid-based Mindtool which helps the students to organize and share what they learned during the game playing. To evaluate the performance of science students on the learning environment a test is performed which shows that the learning performance of the students is increased if they spend more time on discussion and organizing the knowledge. A game-based learning (GBL) framework was proposed in [16] for elementary school students which proved to be more effective than the ordinary textual web pages.

As far as training is concerned, [17] combined 2D and $3 \mathrm{D}$ virtual reality (Virtual Reality Trainers) to provide virtual training to soldiers. The use of Virtual Reality Trainers proved to be cost effective and flexible method of providing training by allowing students to train at their own pace and time. Similarly, for cardiac training, the Heart Murmur Sim environment provides a system where patients can virtually visit and get assisted in diagnosis of heart-related issues through listening to their cardiac rhythms [18]. Emergency workers for UC Davis Medical Center are trained using the virtual environment in a simulated context to act in emergency situation before acting in a real world situation.

To teach about the culture of China a web-based text and multimedia project named second China is developed using Second Life environment. The second China provides an opportunity to explore different cultural activities [19]. Another project named the Tactical Language and Culture Training System (TLCTS) is developed to teach functional skills in four different languages of Iraqi, Dari, Pashto, and French by [20]. There are three different modes of interaction in TLCTS which provide different kinds of learning activities which are skill builder, arcade, and mission games.

The existing technoliteracy solutions are generally categorized as desktop application containing lectures and video content as well as a web-based application (2D text-based instructional programs and games). These solutions ignored traditional learning theories such as self-presence, social presence, and situated learning. To address these issues, [21] unified traditional learning theories three-dimensional (3D) technologies by proposing a learning platform based on Multiple Intelligences (MI) Theory. The authors utilized users' familiar objects for teaching the alphabets to adult absolute illiterate. Basically, the objects are related with the alphabets; for example, the symbol of a famous TV channel is connected with the corresponding alphabets in this work. Although the environment proved to be good for adult absolute illiterate; however, it ignored the concept of sense of belonging.

According to "Maslow's hierarchy of needs" belonging is one of the important factors known as "emotional need" [9] as shown in Figure 1. Belonging means acceptance as a member or part. A sense of belonging is a human need, just like the need for food and shelter. Sense of belonging is associated 


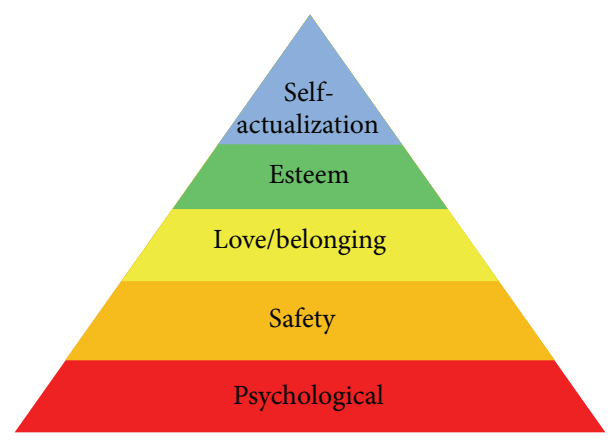

FIGURE 1: Maslow's hierarchy of needs [9].

with harmony between an individual and the surrounding environment. It is developed in the space of conditions for an individual's self-realization that balances individual and social interests [22]. A social relationship providing sense of belonging is more meaningful for learners [23]. This view is also evident, for example, in [24-27] as well, and carries more motivation for learners [28]. It is human nature that we feel more comfortable in the environment which is attractive for us; for instance, if a person likes nature then he would feel more interested in the natural environment. Some people find belonging with friends and family and some on Twitter or other social media.

Our main contribution is how we classify users of the application according to their interests and in designing of learning environments according to user classification. The objects and environment both are of the users' interest. We are adding in existing knowledge of the user by concept of cognitive learning and sense of belonging. While the previous work ignored the concept of interest/belonging and considered only the object that is known by user, however, these objects may not be of the users' interest.

\section{Research Methodology}

This section elaborates the methodology utilized in this work to design a $3 \mathrm{D}$ virtual learning environment for absolute adult illiterate. A focus group based study was conducted in order to get users' interest. The farmers' interests are collected by making a visit to their environment while women's interests are gathered by a focus group study.

3.1. Participants. Participants of our study were adult absolute learners (people who are unable to read or write the numbers). However, all of the participants were able to identify local currency notes by their colour or presentation of currency note. In addition to this, most of the participants are able to do shopping by using currency notes and are capable of calculating the remaining amount easily.

3.2. Participants' Classification. To find the interest and belongings of adult learners, learners are divided into two groups according to gender, namely, males and females. From male class, we selected farmers and, from the female participants, housewives were selected since mostly/predominantly adult illiterate females were housewives.

3.3. Real Environment Visit. A visit to real working environment of farmers is performed in order to design the learning environment. The purpose of this visit was to observe the working environment of farmers and to map real environment objects into the 3D learning environment. The visit was conducted to collect the interest of farmers. We coordinated with farmers and team members to plan our visits. The team consisted of expert from areas of human computer interaction, software requirement engineers, virtual reality developer, and educationist.

3.4. Finding Interests and Belongings of Farmers. Team members observed real world environment and its different objects. The importance of the objects was investigated by the team from farmers. A list of significant objects according to farmers was noted down. Pictures of these significant objects and the environment were captured for our record to map them into the $3 \mathrm{D}$ environment. After returning from real environment visit, next day team members discussed the listed objects from different prospects; for instance, education, mapping, human computer interaction, and 18 vital objects of farmer environment were selected.

3.5. Focus Group Study to Find Interest and Belonging of Women. To find the interests of women, 17 women were requested to list down 15 different objects of their interests. The restriction about the object selection was that the objects must be very common and everyone could identify these objects. After getting the list of objects from women, the colour printing of these objects was performed. The prints were given to 38 illiterate women and the women were asked to assign a number ranging from 1 to 15 to the objects based on their importance ( 1 for the most important and 15 for the least important). After filtering, 10 most common objects were chosen to model in the $3 \mathrm{D}$ environment.

3.6. Icon Selection. To map selected object into the $3 \mathrm{D}$ environment, icons (images) for the selected objects were downloaded. It is difficult to select an icon for a selected object as a number of icons (images) were downloaded for a single object. Most related icons were identified and provided to a team of five farmers. The team finalized the icons for the selected objects. A similar approach was adopted to finalize the icons for women. For generalized environment, we followed the methodology adopted by [21] to finalize the objects in which commonly known objects are selected; however, the environment does not contain the objects of pedagogy. This environment is designed without focusing any specific group of learners. The design of the $3 \mathrm{D}$ environment is based on the pictures of real world environment that we took during our visit to real world environment. The real effect of environment makes learning environment more attractive for learners due to their interests and familiarity. 


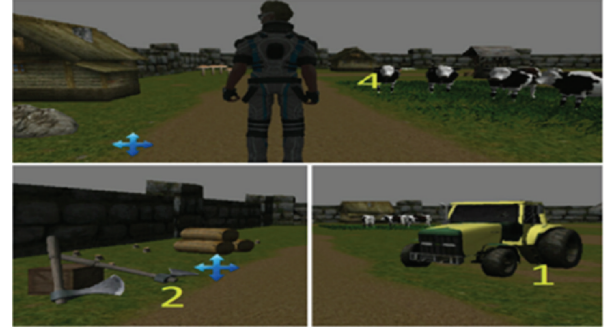

FIGURE 2: Virtual learning environment for farmers showing numeracy education.

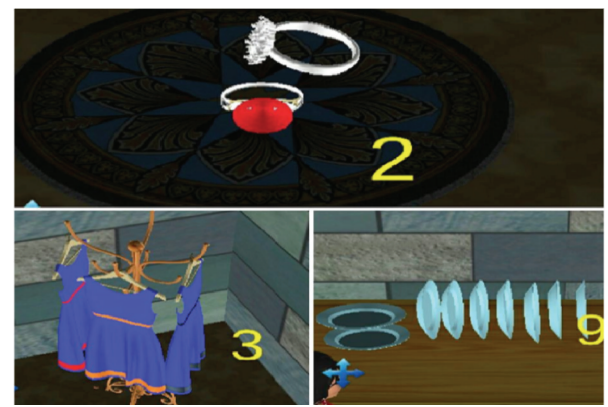

FIGURE 3: Virtual learning environment for women showing numeracy education.

\section{Virtual Learning Environment for Adult Absolute Illiterates}

Three types of environment, namely, a general environment, environment for farmers, and environments for women, are modelled in the study based on the sense of belonging. The user interface for such environments is developed using unity 3D engine (https://unity3d.com/). The user interface of the environment is designed in NGUI 3.0. C\# scripting language is used to control the program activity. We use free asset to develop this game which is downloaded from official unity asset store. Some assets are downloaded from online resources (http://www.archive3d.net/). Few of the assets are designed in 3D animation making tool Blender. After successfully testing our system using unity emulator, we also tested the environments on multiple devices. Figures 2 and 3 show the proposed environment for farmers and women.

\section{Evaluation of Proposed Users' Centered Environment}

We conducted experiments with farmers and women to access the effectiveness of the proposed environment. A total of 120 learners (60 farmers and 60 women) participated in the experiment. Farmers and women were divided into control (30 farmers and 30 women) and experimental (30 farmers and 30 women) groups. The generalized environment was provided to the control group whereas users' centered environments were provided to experimental group. One hour was given to both groups to learn the numeracy from
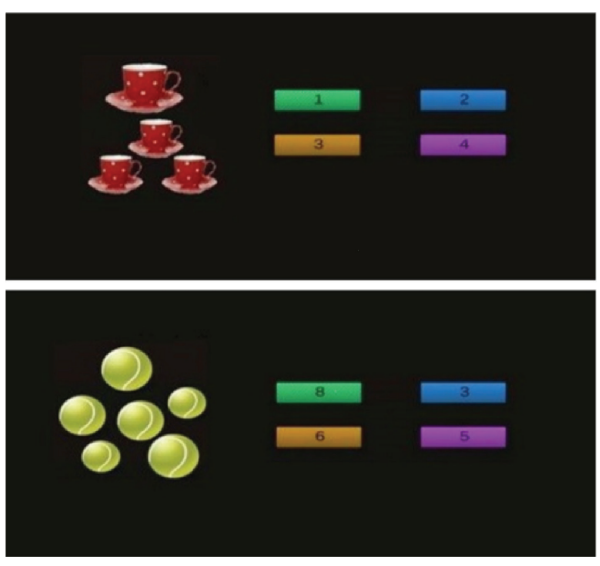

FIgURE 4: Quiz application.

0 to 9 using the prototype. In the prototype, when the animated agent (learner) passes from the objects then the numbers of objects appear on the screen (Figures 2 and 3 ), and agent speaks that number in local language (Urdu language). The user can also count the number of objects to verify and memorize the symbol used for that number. After one hour, a quiz was conducted with the participants to access their learning efficiency as shown in Figure 4. On selecting the correct answer the learner gains 1 mark and learning is measured by number of correct answers; for example, if learners recognized 6 digits correctly out of ten ( 0 to 9$)$ then his learning efficiency was calculated as $60 \%$. It is pertinent to mention that both the control and the experimental group take the same test. After conducting the quiz, the learning efficiency of each learner was recorded.

In order to evaluate the effectiveness of the proposed users' centered environment, an independent sample $t$-test was conducted. The numeracy learning efficacy of farmers and women using generalized and users' centered environment is compared. We considered the general and users' centered environment as independent variable and the accuracy of learning as dependent variables. There was a significant difference in learning efficiency for general environment $(M=61.00, \mathrm{SD}=13.80)$ and users' centered environment $(M=74.66, \mathrm{SD}=15.15 ; t(27)=-2.538, p=.008$, twotailed) as reported by farmers. Farmers exhibited improved learning output on users' centered environment as compared to general environment. Similarly, significant difference was found in learning efficacy of women for general $(M=54.00$, $\mathrm{SD}=9.856)$ and users' centered environment $(M=65.33$, $\mathrm{SD}=12.46 ; t(28)=2.763, p=.010$, two-tailed). This is also summarized in Table 1. Better numeracy learning efficiency was reported on users' centered environment by women. Likewise, significant difference was identified between computer based environment $(M=55.00, \mathrm{SD}=10.09)$ and mobile based environment $(M=66.33, \mathrm{SD}=12.45 ; t(28)=$ $-2.835, p=.012$, two-tailed). It is important to highlight that mobile based learning environment was more useful as already reported in [29-32].

Finally, we conducted semistructured interview to see if a mobile based application is more useful than a traditional 
TABLE 1: Difference in numeracy learning efficiency between general and users' centered environment.

\begin{tabular}{|c|c|c|c|c|c|}
\hline Participant & Environment & Mean & SD & $t$ value & $p$ value \\
\hline \multirow{2}{*}{ Farmers } & General environment & 57.33 & 12.23 & \multirow{2}{*}{-2.628} & \multirow{2}{*}{.009} \\
\hline & Users' centered environment & 69.33 & 11.00 & & \\
\hline \multirow{2}{*}{ Women } & General environment & 65.33 & 12.46 & \multirow{2}{*}{2.763} & \multirow{2}{*}{.010} \\
\hline & Users' centered environment & 54.00 & 9.856 & & \\
\hline \multirow{2}{*}{ Technology } & Computer based learning environment & 63.33 & 11.75 & \multirow{2}{*}{-2.628} & \multirow{2}{*}{.014} \\
\hline & Mobile based learning environment & 74.67 & 11.87 & & \\
\hline
\end{tabular}

PC based application. We found out that a mobile application was more useful in many ways and participants noted the following key reason for this:

(a) It was easy to understand the content since it was related to their everyday life and the environment that they were used to.

(b) Users liked local language support since it augmented their learning process.

(c) Mobile based system provided them freedom and there were no time restrictions and they could use the application whenever they were free.

(d) Finally, the target user group, that is, absolute illiterates, were not familiar with use of personal computers.

Participants highlighted that PC based system was not a practical idea in their case due to the fact that there were electrical power related issues in their households.

\section{Conclusion}

The existing learning solutions are mostly for toddler or functional literate adults. Further, these solutions ignored learners' style, cognitive learning, and sense of belonging. This paper addresses these discrepancies by proposing users' centered 3D learning environments for farmers and women adult learners. To make the environment attractive and effective and to incorporate sense of belonging, data was collected from farmers and women. Then the collected data incorporated in the environments in order to improve the numeracy education of adult absolute learners. Moreover, we also conducted an experimental study to investigate the impact of users' centered design on the learning efficiency of adults and the results spot-lighted a strong impact of users' centered design on adult learning. As a next step, we plan to develop a 2D user-centered environment and compare it with our work presented in this paper to evaluate which of these two would be best suited to augment learning at the level of our target audience.

\section{Conflict of Interests}

The authors declare that there is no conflict of interests regarding the publication of this paper.

\section{References}

[1] V. N. Parrillo, Encyclopedia of Social Problems Illiteracy, Adult in Developing Nations, SAGE Knowledge, 2008.

[2] M. B. Horn and H. C. Staker, The Rise of K12 Blended Learning, Innosight Institute, Chapel Hill, NC, USA, 2011.

[3] UNESCO Report, March 2013, http://www.unesco.org/new/n/ media-services/single-view/news/8_september_international_literacy_day_793_million_adults_can_neither_read_nor_write/.

[4] M. Richmond, C. Robinson, and M. Sachs-Israel, The Global Literacy Challenge: A Profile of Youth and Adult Literacy at the Mid-Point of the United Nations Literacy Decade 2003-2012, United Nations Educational Scientific and Cultural Organization, UNESCO, 2008.

[5] J. Sabatini, "Adult reading acquisition," in Literacy: An International Handbook, D. A. Wagner, R. L. Venezky and, and B. L. Street, Eds., Westview Press, Boulder, Colo, USA, 1999.

[6] U. J. Nwosu, How Does Technology Impact on the Self-beliefs of Adult Basic Education Learners? Lambert Academic Publishing, Saarbrücken, Germany, 2010.

[7] M. D. Dickey, "Brave (new) interactive worlds: a review of the design affordances and constraints of two 3D virtual worlds as interactive learning environments," Interactive Learning Environments, vol. 13, no. 1-2, pp. 121-137, 2005.

[8] S. Robbins-Bell, "Higher education as virtual conversation," EDUCAUSE Review, vol. 43, no. 5, pp. 24-35, 2008.

[9] A. H. Maslow, "A theory of human motivation," Psychological Review, vol. 50, no. 4, pp. 370-396, 1943, http://psychclassics .yorku.ca/Maslow/motivation.htm.

[10] J. C. Yang, C. H. Chen, and M. C. Jeng, "Integrating videocapture virtual reality technology into a physically interactive learning environment for English learning," Computers and Education, vol. 55, no. 3, pp. 1346-1356, 2010.

[11] N. Lambropoulos and S. Mystakidis, "Learning experience+ within 3D immersive worlds," in Proceedings of the Federated Conference on Computer Science and Information Systems (FedCSIS '12), pp. 857-862, September 2012.

[12] N. Adamo-Villani, E. Carpenter, and L. Arns, "3D sign language mathematics in immersive environment," in Proceedings of the Special Interest Group on Computer Graphics and Interactive Techniques Conference (SIGGRAPH '06), ACM, Rhodes, Greece, August 2006.

[13] L. Daghestani, R. D. Ward, Z. Xu, and H. Al-Nuaim, "The design, development and evaluation of virtual reality learning environment for numeracy concepts using 3D virtual manipulatives," in Proceedings of the 5th International Conference on Computer Graphics, Imaging and Visualisation (CGIV '08), M. Sarfraz, E. Banissi, and W. C. Jeng, Eds., pp. 93-100, IEEE Computer Society, Penang, Malaysia, August 2008. 
[14] J. C. Lester, H. A. Spires, J. L. Nietfeld, J. Minogue, B. W. Mott, and E. V. Lobene, "Designing game-based learning environments for elementary science education: a narrativecentered learning perspective," Information Sciences, vol. 264, pp. 4-18, 2014.

[15] H.-Y. Sung and G.-J. Hwang, "A collaborative game-based learning approach to improving students' learning performance in science courses," Computers and Education, vol. 63, pp. 43-51, 2013.

[16] C.-S. Wang, C.-C. Liu, and Y.-C. Li, "A game-based learning content design framework for the elementary school children education," in Proceedings of the 15th North-East Asia Symposium on Nano, Information Technology and Reliability (NASNIT '11), pp. 53-57, IEEE, Macau, China, October 2011.

[17] L. R. McMaster, G. E. Cooper, D. M. McLin, D. L. Field, R. L. Baumgart, and G. A. Frank, "Combining 2D and 3D virtual reality for improved learning," in Proceedings of the Interservice/Industry Training, Simulation and Education Conference (I/ITSEC '02), 2002.

[18] S. FitzGerald and J. Kay, N. D. Second Life in Education, November, 2008, http://sleducation.wikispaces.com.

[19] J. Henderson, P. Fishwick, E. Fresh, F. Futterknecht, and B. D. Hamilton, "Immersive learning simulation environment for chinese culture," in Proceedings of the Interservice/Industry Training, Simulation, and Education Conference, Paper 8334, 2008.

[20] W. L. Johnson and A. Valente, “Tactical Language and Culture Training Systems: using artificial intelligence to teach foreign languages and cultures," in Proceedings of the 20th Inational Conference on Innovative Applications of Artificial Intelligence (IAAI '08), vol. 3, pp. 1632-1639, AAAI Press.

[21] T. Iqbal, K. Hammer Müller, and A. Tjoa, "Second life for illiterates: a 3D virtual world platform for adult basic education," in Proceedings of the 12th International Conference on Information Integration and Web-Based Applications and Services (iiWAS '10), pp. 371-378, Paris, France, November 2010.

[22] O. Rode, "Educational and psychological aspects of environmental awareness and a sense of belonging," Discourse and Communication for Sustainable Education, vol. 4, no. 1, pp. 6779, 2015.

[23] N. M. Lambert, T. F. Stillman, J. A. Hicks, S. Kamble, R. F. Baumeister, and F. D. Fincham, "To belong is to matter: sense of belonging enhances meaning in life," Personality and Social Psychology Bulletin, vol. 39, no. 11, pp. 1418-1427, 2013.

[24] J. P. Connell and J. G. Wellborn, "Competence, autonomy, and relatedness: a motivational analysis of self-system processes," The Minnesota Symposia on Child Development: Self-Processes and Development, vol. 23, pp. 43-77, 1991.

[25] E. L. Deci and R. M. Ryan, "A motivational approach to self: integration in personality", in Proceedings of the Nebraska Symposium on Motivation: Perspectives on Motivation, vol. 38, pp. 237-288, Lincoln, Neb, USA, 1991.

[26] J. D. Finn, "Withdrawing from school," Review of Educational Research, vol. 59, no. 2, pp. 117-142, 1989.

[27] K. F. Osterman, "Students' need for belonging in the school community," Review of Educational Research, vol. 70, no. 3, pp. 323-367, 2000.

[28] C. Goodenow, “The psychological sense of school membership among adolescents: scale development and educational correlates," Psychology in the Schools, vol. 30, no. 1, pp. 79-90, 1993.

[29] A. T. Korucu and A. Alkan, "Differences between m-learning (mobile learning) and e-learning, basic terminology and usage of m-learning in education," Procedia-Social and Behavioral Sciences, vol. 15, pp. 1925-1930, 2011.

[30] Y. L. Y. Liu, J. L. J. Liu, and S. Y. S. Yu, "A case study on mobile learning implementation in basic education," in Proceedings of the IEEE International Conference on Computer Science and Software Engineering, vol. 5, pp. 593-597, Wuhan, China, December 2008.

[31] K. J. Lee and J. E. Kim, "A mobile-based learning tool to improve writing skills of EFL learners," Procedia-Social and Behavioral Sciences, vol. 106, pp. 112-119, 2013.

[32] J.-H. Valk, A. T. Rashid, and L. Elder, "Using mobile phones to improve educational outcomes: an analysis of evidence from Asia," International Review of Research in Open and Distance Learning, vol. 11, no. 1, pp. 117-140, 2010. 

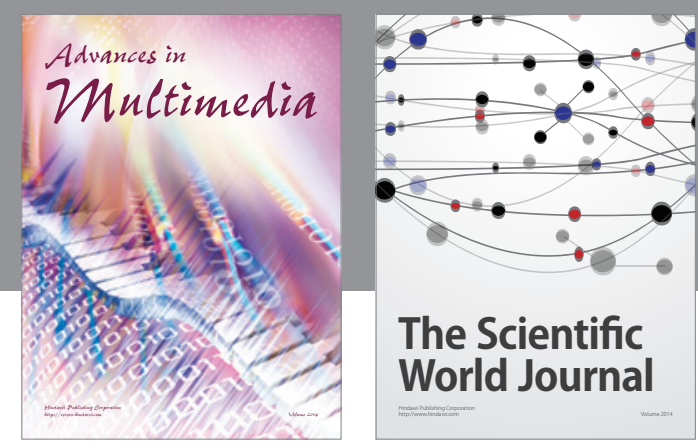

The Scientific World Journal
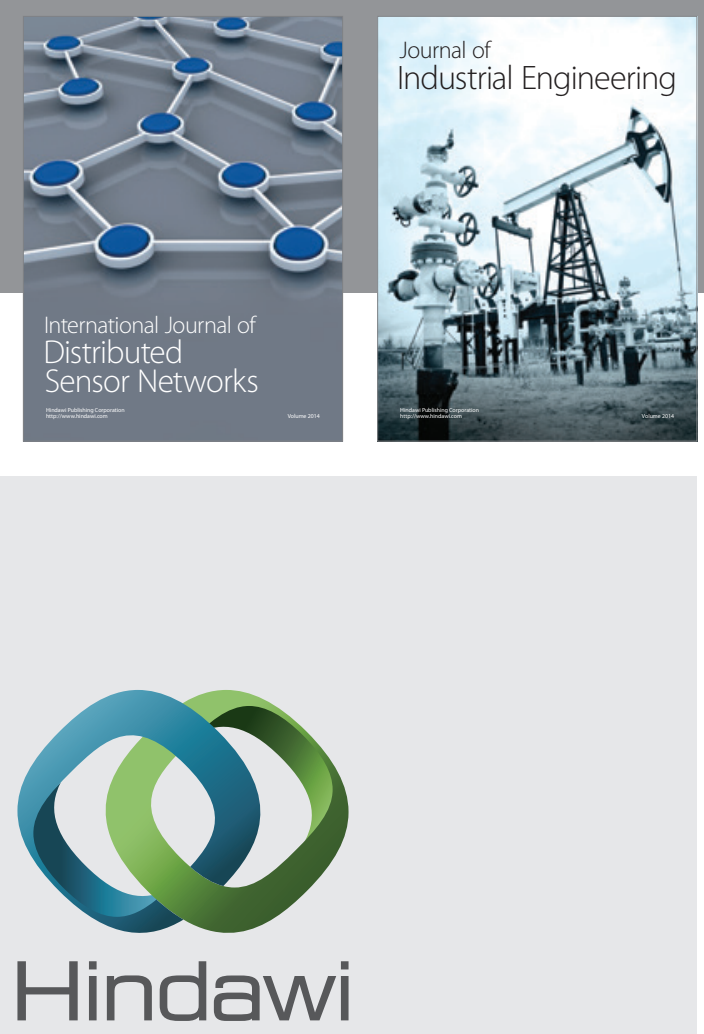

Submit your manuscripts at

http://www.hindawi.com

\section{Computer Networks} and Communications
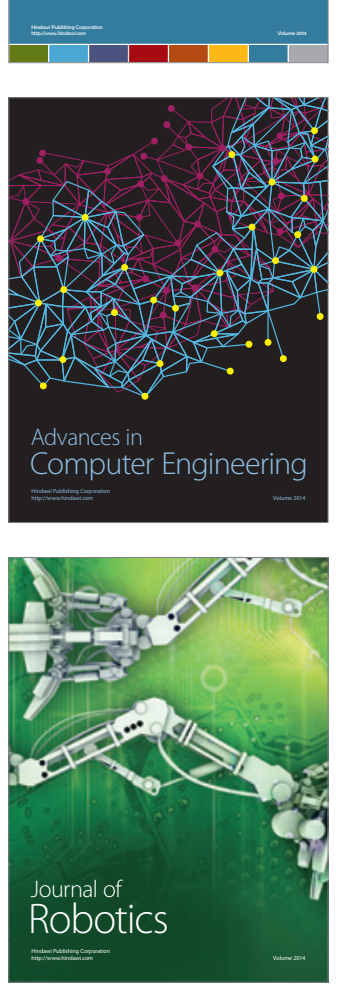
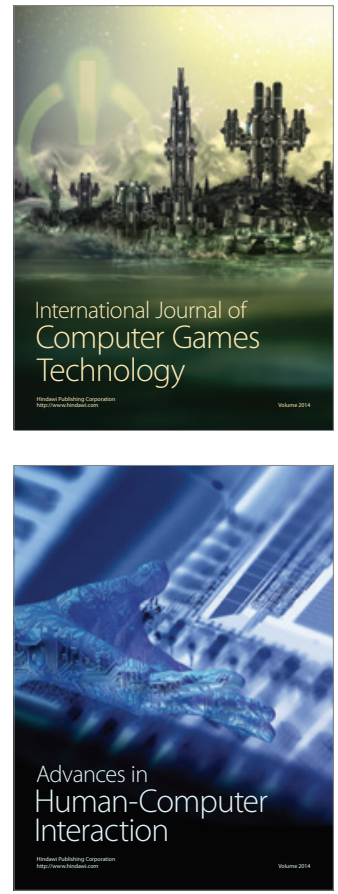
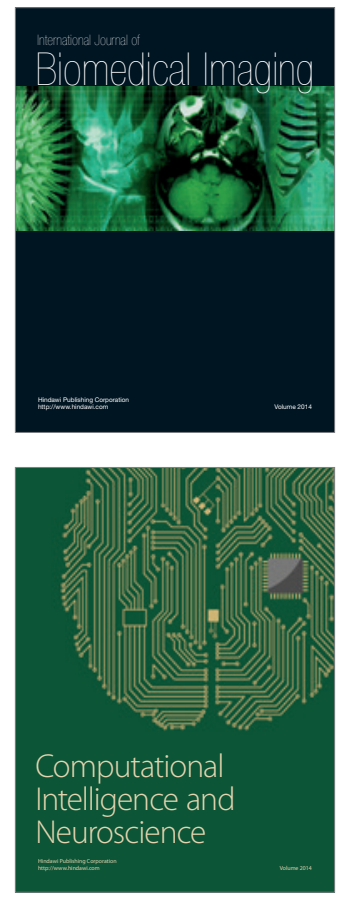
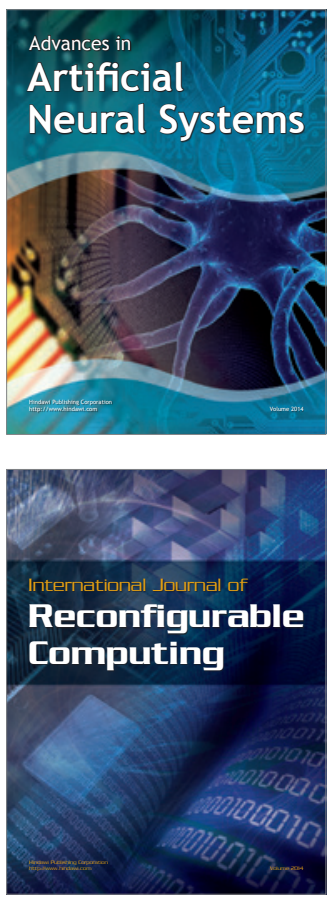
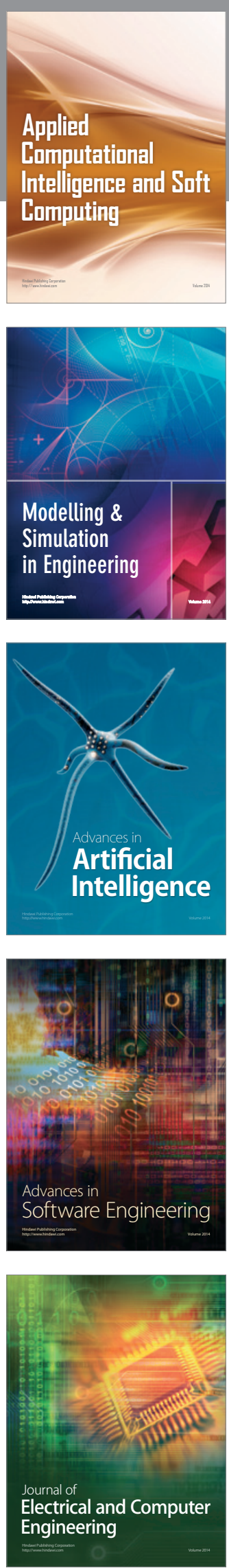